\title{
Segment-Based Acoustic Models for Continuous Speech Recognition
}

\author{
Mari Ostendorf J. Robin Rohlicek \\ Boston University \\ Boston, MA 02215 \\ BBN Inc. \\ Cambridge, MA 02138
}

\section{PROJECT GOALS}

The goal of this project is to develop improved acoustic models for speaker-independent recognition of continuous speech, together with efficient search algorithms appropriate for use with these models. The current work on acoustic modeling is focussed on stochastic, segmentbased models that capture the time correlation of a sequence of observations (feature vectors) that correspond to a phoneme, hierarchical stochastic models that capture higher level intra-utterance correlation, and multipass search algorithms for implementing these more complex models. This research has been jointly sponsored by DARPA and NSF under NSF grant IRI-8902124 and by DARPA and ONR under ONR grant N00014-92-J-1778.

\section{RECENT RESULTS}

- Implemented different auditory-based signal processing algorithms and evaluated their use in recognition on the TIMIT corpus, finding no performance gains relative to cepstral parameters probably due to the non-Gaussian nature of auditory features.

- Improved the score combination technique for $\mathrm{N}$ Best rescoring, through normalizing scores by sentence length to obtain more robust weights that alleviate problems associated with test set mismatch.

- Further investigated agglomerative and divisive clustering methods for estimating robust contextdependent models, and introduced a new clustering criterion based on a likelihood ratio test; obtained a slight improvement in performance with an associated reduction in storage costs of a factor of two.

- Extended the classification and segmentation scoring formalism to handle context-dependent models without requiring the assumption of independence of features between phone segments (using maximum entropy methods); evaluated different segmentation scores with results suggesting more work is needed in this area.

- Evaluated a new distribution mapping, which led to an $8 \%$ reduction in error on the development test set but no improvement on other test sets.

- Investigated the use of different phone sets and probabilistic multiple-pronunciation networks; no improvements were obtained on the RM corpus, though there may be gains in another domain.

- Extended the two level segment/microsegment formalism to application in word recognition using context-dependent models; evaluated the trade-offs associated with modeling trajectories vs. (non-tied) microsegment mixtures, finding that mixtures are more useful for context-independent modeling but representation of a trajectory is more useful for context-dependent modeling.

- Investigated the use of tied mixtures at the frame level (as opposed to the microsegment level), evaluating different covariance assumptions and training conditions; developed new, faster mixture training algorithms; and achieved a $20 \%$ reduction in word error over our previous best results on the Resource Management task. Current SSM performance rates are $3.6 \%$ word error on the Oct 89 test set and $7.3 \%$ word error on the Sep92 test set.

\section{PLANS FOR THE COMING YEAR}

- Continue work in the classification and segmentation scoring paradigm; demonstrate improvements associated with novel models and/or features.

- Port the BU recognition system to the Wall Street Journal (WSJ) task, 5000 word vocabulary.

- Develop a stochastic formalism for modeling intrautterance dependencies assuming \& hierarchical structure.

- Investigate unsupervised adaptation in the WSJ task domain.

- Investigate multi-pass search algorithms that use a lattice rather than $\mathrm{N}$-Best representation of recognition hypotheses. 